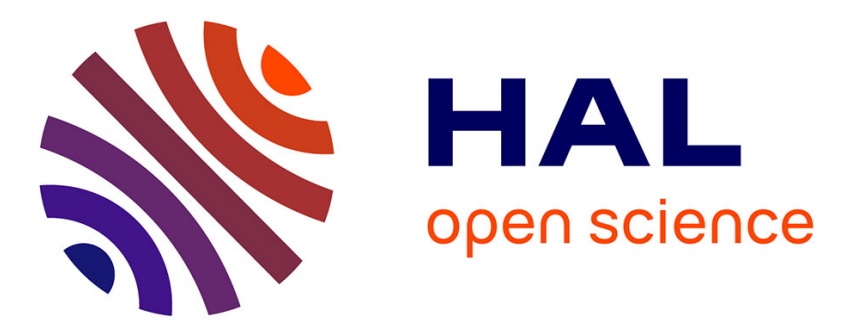

\title{
CloudaSec: a novel public-key based framework to handle data sharing security in clouds
}

\author{
Nesrine Kaaniche, Maryline Laurent, Mohammed El Barbori
}

\section{To cite this version:}

Nesrine Kaaniche, Maryline Laurent, Mohammed El Barbori. CloudaSec: a novel public-key based framework to handle data sharing security in clouds. SECRYPT 2014: 11th International Conference on Security and Cryptography, Aug 2014, Vienne, Austria. pp.5 - 18, 10.5220/0005010600050018 . hal-01263351

\section{HAL Id: hal-01263351 \\ https://hal.science/hal-01263351}

Submitted on 27 Jan 2016

HAL is a multi-disciplinary open access archive for the deposit and dissemination of scientific research documents, whether they are published or not. The documents may come from teaching and research institutions in France or abroad, or from public or private research centers.
L'archive ouverte pluridisciplinaire HAL, est destinée au dépôt et à la diffusion de documents scientifiques de niveau recherche, publiés ou non, émanant des établissements d'enseignement et de recherche français ou étrangers, des laboratoires publics ou privés. 


\title{
CloudaSec: A Novel Public-key based Framework to handle Data Sharing Security in Clouds
}

\author{
Nesrine Kaaniche ${ }^{1}$, Maryline Laurent ${ }^{1}$, Mohammed El Barbori ${ }^{1}$ \\ ${ }^{1}$ Institute Mines Telecom, Telecom-SudParis \\ \{nesrine.kaaniche, maryline.laurent, mohammed.el_barbori\}@telecom-sudparis.eu
}

\begin{abstract}
Keywords: cloud storage systems, data security, access control, algorithm verification
Abstract: Recent years have witnessed the trend of leveraging cloud-based services for large scale content storage, processing, and distribution. Data security and privacy are among top concerns for the public cloud environments. Towards these security challenges, we propose and implement CloudaSec framework for securely sharing outsourced data via the public cloud. CloudaSec ensures the confidentiality of content in the public cloud environments with flexible access control policies for subscribers and efficient revocation mechanisms. CloudaSec proposes several cryptographic tools for data owners, based on a novel content hash keying system, by leveraging the Elliptic Curve Cryptography (ECC). The separation of subscription-based key management and confidentiality-oriented asymmetric encryption policies uniquely enables flexible and scalable deployment of the solution as well as strong security for outsourced data in cloud servers. Through experimental evaluation, we demonstrate the efficiency and scalability of CloudaSec, build upon OpenStack Swift testbed.
\end{abstract}

\section{INTRODUCTION}

Recently, the US International Data Corporation (IDC) proclaims that the digital universe will grow by a factor of 300 , up to 40 trillion gigabytes of replicated data by 2020 (Gantz and Reinsel, 2012). This explosive growth of data continues to rise the demand for new storage and network capacities, along with an increasing need for more cost effective architectures. As such, recent years have witnessed the trend of leveraging cloud data storage, since it provides efficient remote storage services in pay per use business model.

However, these promising data storage services have brought many challenging design issues, considerably due to the loss of control on outsourced data. One of the biggest concerns is data confidentiality provisioning which remains a fundamental security requirement in cloud storage services.

It is commonly agreed that data encryption at the client side is a good alternative to mitigate such concerns of data confidentiality. Thus, the client preserves the decrypting keys out of reach of the cloud provider. Nonetheless, the confidentiality preservation becomes more complicated with flexible data sharing among a group of users. First, it requires efficient sharing of decrypting keys between different authorized users. The challenge is to define a smooth group revocation which does not require updating the secret keys of the remaining users. So, the complexity of key management is minimized. Second, the access control policies should be flexible and distinguishable among users with different privileges to access data. That is, data may be shared by different users or groups, and users may belong to several groups.

In this paper, we propose CloudaSec, a public key based solution for improving data confidentiality in cloud storage environments and enhancing dynamic sharing between users. CloudaSec applies the convergent encryption concept (Wang et al., 2010) on data contents. That is, the data owner uploads encrypted content to the cloud and seamlessly integrates the deciphering key encrypted into the metadata to ensure data confidentiality. In addition, CloudaSec integrates a conference key distribution scheme, based on parallel Diffie Hellman exchanges, in order to guarantee backward and forward secrecy (Burmester and Desmedt, 2005). That is, only authorized users can access metadata and decipher the decrypting data keys. As such, user revocation is achieved without updating the private keys of the remaining users.

Beyond these security properties, a deduplication mechanism is deployed ensuring that only one copy of content is stored in cloud servers. This feature enables the efficient usage of storage capacities and achieves fast data distribution. 
Paper Organization- The remainder of this work is organized as follows: Section 2 presents security considerations and design goals. Then, Section 3 describes the system model, reviews some preliminaries and cryptographic primitives, details the framework design, and describes the prototype and its different procedures. In Section 4, rigorous security discussions are given, and implementation results are discussed in Section 5. Finally, we review the related work in Section 6, before concluding in Section 7.

\section{PROBLEM STATEMENT}

Providing data confidentiality, in multi-tenant environments, becomes more challenging and conflicting. This is largely due to the fact that users outsource their data on remote servers, which are controlled and managed by possible untrusted Cloud Service Providers (CSPs). That is why, it is compulsory to provide secrecy by encrypting data before their storage in cloud servers while keeping the decryption keys out of reach of CSP and any malicious user. Nonetheless, the confidentiality preservation becomes more complex with resilient data sharing among dynamic groups. Hence, secure data sharing should support flexible security policies including forward and backward secrecy.

- Forward secrecy - this property requires that the confidentiality of previous encrypted data has to be ensured even after the long-term secrets are exposed. For example, a user cannot access stored data before he joins a group.

- Backward secrecy - this property means that a compromise of the secret key does not affect the secrecy of future encrypted data. As such, a revoked group member is unable to access data that were outsourced after he leaves the group.

Therefore, the design of our protocol is motivated by providing the support of both robustness and efficiency, while considering the limited storage and processing capacities of user devices. It has to fulfill the following requirements:

- Data confidentiality - our scheme has to protect the secrecy of outsourced data contents against both curious providers and malicious users.

- Flexible access control - CloudaSec should ensure flexible security policies among users with different granted privileges, belonging to different groups. These access control policies should guarantee backward and forward secrecy of outsourced data contents.

- Efficient user revocation - the revocation of a group member should not affect the remaining users. That is, contrary to traditional fine-grained access control schemes, the challenge is to define a smooth group revocation which does not requires updating the secret keys of the non-revoked members.

- Low computation overhead - on one hand, for scalability reasons, the amount of computation at the cloud storage server should be minimized, as the server may be involved in concurrent interactions. On the other hand, the proposed algorithms should also have low processing complexity, at the client side.

- Low communication overhead - CloudaSec should minimize the usage of bandwidth, relying on low communication cost.

- Low storage cost - the limited storage capacities of the user devices has a critical importance in designing our solution. So, low storage cost at the client side is highly recommended.

\section{CLOUDASEC FRAMEWORK}

This section presents CloudaSec architecture with four different types of players. Then, it introduces CloudaSec, a public key based framework to handle data sharing security, and it highlights the cryptographic assumptions that should be fulfilled by our proposed framework.

\subsection{System Model}

Figure 1 illustrates a descriptive network architecture for CloudaSec framework. It relies on the following entities for the good management of client data:

- Cloud Service Provider (CSP): a CSP has significant resources to govern distributed cloud storage servers and to manage its database servers. It also provides virtual infrastructure to host application services. These services can be used by the client to manage his data stored in the cloud servers.

- Data owner: a data owner makes use of provider's resources to store, retrieve and share data with multiple users. A data owner can be either an individual or an enterprise.

- Group Manager (GM): a group manager takes charge of construction of a group, system parameters generation, user registration and user revocation. Therefore, we assume that the group manager is trusted by the other entities.

- Users: the users are able to access the content stored in the cloud, depending on their access 
rights which are authorizations granted by the data owner, like the rights to read, write or re-store the modified data in the cloud. These access rights serve to specify several groups of users.

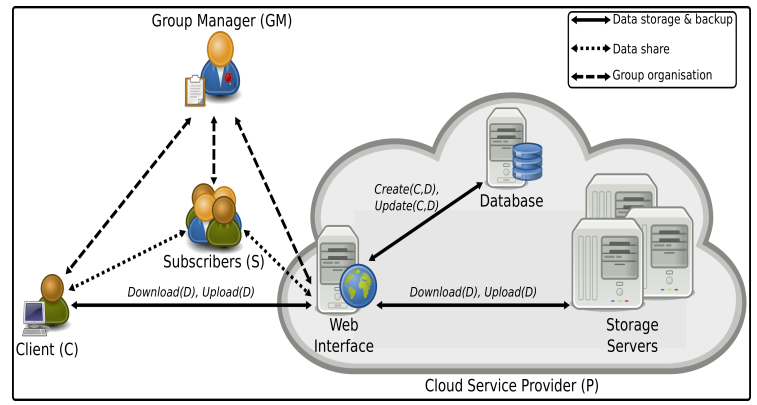

Figure 1: CloudaSec architecture

In practice, the CSP provides a web interface for data depositors to store data into a set of cloud servers, which are running in a cooperated and distributed manner. In addition, the web interface is used by the users to retrieve, modify and re-store data from the cloud, depending on their access rights. We assume that there is an established secure channel between the cloud user and the CSP. This secure channel supports mutual authentication and data confidentiality and integrity. It can be implemented through the Transport Layer Security protocol (TLS) (Dierks and Rescorla, 2008), where the client can authenticate with a certificate or password.

Next, we refer to these authorized user(s) as the recipient(s) and to the data owner as the depositor. We must note that our proposal does not require from the recipients to be connected during the sharing process. Indeed, recipients' access rights are granted by the data owner and managed by the CSP. That is, the CSP is in charge of verifying each recipient access permissions before sending him a redirected access key element.

\subsection{CloudaSec Overview}

To protect outsourced data in public cloud servers from unauthorized entities, CloudaSec provides several cryptographic tools for the data owner in order to guarantee the secrecy of his outsourced data and to ensure that only authorized users are able to obtain the decrypting data keys.

Our framework relies on the convergent encryption (Wang et al., 2010) which is a content hash keying cryptographic system. That is, it presents two encryption levels: data encryption level and key encryption level as follows.

- symmetric data encryption level - before out- sourcing data to cloud servers, the depositor encrypts file contents, using a symmetric algorithm. That is, the enciphering data key is derived, from the file plaintext, using a one way hash function. Hence, the choice of the convergent encryption is multifold. First, storage capacity is preserved as the same data encrypted by several users produce the same encrypted data that need to be stored once. As such, the number of redundant copies is minimized in order to preserve the efficiency of the storage service. Second, convergent encryption leads to a per-data enciphering key thus mitigating the usual key sharing problem when content sharing is needed. Third, the generation of the deciphering data key is possible only if the plaintext is known.

- asymmetric key encryption level - the depositor enciphers the decrypting data key $k$, based on an asymmetric algorithm, using the public key of the recipient. Then, he includes this resulted encrypted key in user metadata, ensuring flexible access policies. Indeed, any authorized recipient may access to user metadata, in order to decipher the encrypted data key, using his private key. Then, he can decrypt the enciphered contents.

This dual encryption scheme on data then on the decrypting keys provides data confidentiality, as well as flexible access control policies.

CloudaSec procedures involve two joint layers: data layer and management layer. In the data layer, we introduce the operations on data and the related enciphering keys, namely GenerateParameters, EncryptData, DecryptData, EncryptKeyOneToOne, EncryptKeyOneToMany and ShrinKey. In the management layer, CloudaSec introduces procedures of user revocation, when a group member leaves or is revoked from the group, and user subscription, when a new user joins the group.

CloudaSec supports flexible access to encrypted contents, by dynamically sharing a group secret key within the group. That is, when the group state is modified due to a user subscription or revocation, the GM broadcasts the new group arrangement to authorized members in order to generate the new secret group key, based on the published public elements, without updating the private keys of the remaining users, as presented in Section 3.5.

CloudaSec distinguishes two different data sharing scenarios. First, the data sharing one to one, presented in Section 3.4.1, where a data owner stores for one CloudaSec user. Second, the data sharing one to many, described in Section 3.4.2, where a data owner shares data among a group of authorized users. These scenarios encompass two different data 
key encryption algorithms EncryptKeyOneToOne and EncryptKey OneToMany.

The different notations used in this paper are listed in Table 1.

Table 1: Our notations

\begin{tabular}{|c|l|}
\hline Notation & Description \\
\hline \hline$f$ & file content \\
\hline$k$ & data key \\
\hline$i d_{i}$ & identity of a CloudaSec user $U_{i}$ \\
\hline$s k_{i}$ & private key of a CloudaSec user $U_{i}$ \\
\hline$p k_{i}$ & public key of a CloudaSec user $U_{i}$ \\
\hline$s k_{c}$ & private key of the CSP \\
\hline$p k_{c}$ & public key of the CSP \\
\hline$d$ & group secret key \\
\hline
\end{tabular}

\subsection{Cryptographic Background}

This section reviews a straightforward cryptographic background, used in the design of our CloudaSec framework.

\subsubsection{Preliminaries}

CloudaSec essentially relies on the use of one way functions and bilinear maps, defined as follows.

Collision resistant hash functions(Boneh and Boyen, 2006) - Let $H:\{0,1\}^{*} \rightarrow\{0,1\}^{n}$ be a hash function. $H$ is a collision resistant function if no efficient algorithm can find a pair $M \neq M^{\prime} \in\{0,1\}^{*}$, such that $H(M)=H\left(M^{\prime}\right)$.

Bilinear maps $\{$ (Regan, ), (Ratna et al., 2004)\} - an admissible symmetric pairing function $\hat{e}$ from $\mathbb{G}_{1} \times \mathbb{G}_{1}$ in $\mathbb{G}_{2}$ has to be bilinear, non degenerate and efficiently computable. $\mathbb{G}_{1}$ is an additive subgroup of the group of points of an Elliptic Curve (EC). However, $\mathbb{G}_{2}$ is a multiplicative subgroup of a finite field. $\mathbb{G}_{1}$ and $\mathbb{G}_{2}$ have the same order $q$. In addition, $\mathbb{G}_{1}$ and $\mathbb{G}_{2}$ are generated by $P$ and the $g=\hat{e}(P, P)$, respectively.

\subsubsection{Cryptographic Assumptions}

Our proposal is based on two cryptographic assumptions, namely the Elliptic Curve Discrete Logarithm Problem and the Computational Diffie Hellman Problem.

Elliptic Curve Discrete Logarithm Problem $(\boldsymbol{E C D L P})$ - given an additive group $\mathbb{G}$, a subgroup of $E\left(\mathbb{F}_{p}\right)$, which is generated by the point $P$ of prime order $n$, it is intractable to find $a$, where $Q=a P$, and $P$ are known.

Computational Diffie Hellman problem $(\mathrm{CDH})-$ given a cyclic group $\mathbb{G}$ of order $p$ and generator $g$, there is no efficient algorithm to calculate $g^{a b}$, where $\left(g, g^{a}, g^{b}\right)$ are known.

\subsubsection{Group Key Distribution (GKD)}

Burmester and Desmedt propose an unauthorized key exchange protocol (Burmester and Desmedt, 2005). It is a two round protocol that extends the concept of the Diffie Hellman assumption.

Let $G=\left\{U_{1}, \ldots U_{m}\right\}$, be a group of $m$ users arranged into a cycle. To generate a group key, each member $U_{i}$, where $i \in[1, m]_{\mathbb{N}}$, first selects a random secret $b_{i}$. Then, he broadcasts $z_{i}=g^{b_{i}}$, where $g$ is a generator of a multiplicative group $\mathbb{G}$. Afterwards, this latter publishes $X_{i}=\left(\frac{z_{i+1}}{z_{i-1}}\right)^{b_{i}}$. We must note that the number of exponentiations per user is constant and each user $U_{i}$ computes $K$, as $K \equiv g^{b_{1} b_{2}+b_{2} b_{3}+\ldots+b_{m} b_{1}} \bmod (p)$.

\subsection{CloudaSec Data Layer Procedures}

This section describes the different CloudaSec data layer procedures. CloudaSec, first, requires a system setup procedure, ensured by the execution of the GenerateParameters algorithm, before performing the sharing scenarios.

This CloudaSec GenerateParameters algorithm initializes the system and generates the public parameters params, according to a required security parameter $\xi$, as presented in Algorithm 1. That is, the system setup procedure generates the groups $\mathbb{G}_{1}$ and $\mathbb{G}_{2}$ and the pairing function $\hat{e}$ from $\mathbb{G}_{1} \times \mathbb{G}_{1}$ in $\mathbb{G}_{2}$. $\mathbb{G}_{1}$ is an additive subgroup of the group of points of an Elliptic Curve (EC), where $\mathbb{G}_{2}$ is a multiplicative subgroup of a finite field. $\mathbb{G}_{1}$ and $\mathbb{G}_{2}$ have the same order $n$ and are generated by $P$ and $g=\hat{e}(P, P)$, respectively.

After the specification of the groups, CloudaSec GenerateParameters procedure defines a secure one way hash function $H: \mathbb{E} \rightarrow\{0,1\}^{l}$, with respect to the required security level, where $\mathbb{E}$ represents the finite data domain and $l$ is the length of the content encrypting key. In addition, it derives an application $F$ to bind an element belonging to the multiplicative group $\mathbb{G}_{2}^{*}$ to a binary sequence of length $l$.

The groups $\mathbb{G}_{1}$ and $\mathbb{G}_{2}$, the pairing $\hat{e}$, the point $P$, the hash function $H()$ and the application $F$ form the public parameters params as follows.

$$
\text { params }=\left\{\mathbb{G}_{1}, \mathbb{G}_{2}, n, \hat{e}, g, P, H(), F\right\} .
$$

We must note that each user has to derive a pair of 


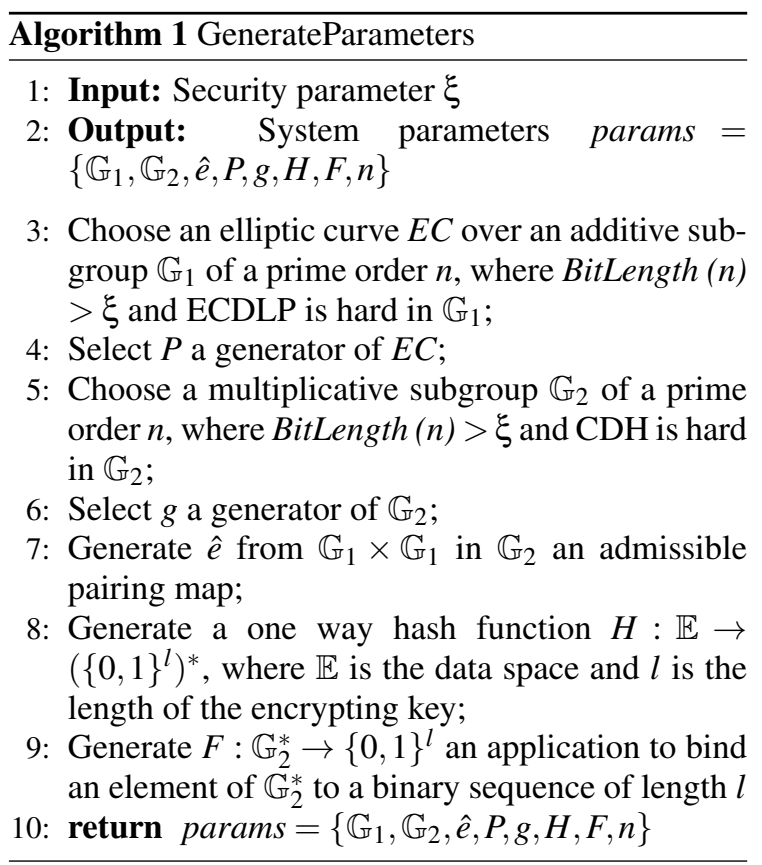

public and private keys, with respect to the published authentic public parameters params and the required security level $\xi$. As such, a CloudaSec user $U_{t}$ is characterized by his identity $i d_{t}$ and the derived pair of keys: his private key $s k_{t}$, where $s k_{t}$ is a random secret $s_{t} \in \in_{R} \mathbb{Z}_{n}$ and his public key as $p k_{t}=s_{t} \cdot P$.

$$
U_{t}\left(i d_{t}, p k_{t}, s k_{t}\right)
$$

In the following, we denote by - the scalar point multiplication in an additive group and by $\star$ two elements multiplication belonging to a multiplicative group.

We consider a data sharing process, where the client outsources his data to the cloud and authorizes a group of users to access the data. This group may be a duo group or a multi-user group.

\subsubsection{CloudaSec One to One Sharing Scenario}

The One to One scenario is defined when a data owner $U_{i}$ wants to share data with only one recipient user $U_{j}$. The depositor $U_{i}$ first enciphers the data file $f$, as presented in Algorithm 2, based on a symmetric encryption scheme SymEnc, using a data enciphering key $k$. Based on a convergent cryptographic solution, the data key $k$ is derived from the application of a one way hash function over the original data file $f$. Subsequently, $U_{i}$ stores the encrypted content $f$ for the recipient user $U_{j}$, in remote servers. In order to assign the access rights to the recipient, the depositor enciphers the data decrypting key $k$ using the public key of the recipient $p k_{j}$, as described in CloudaSec

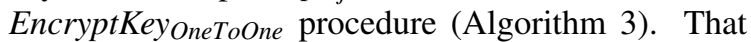

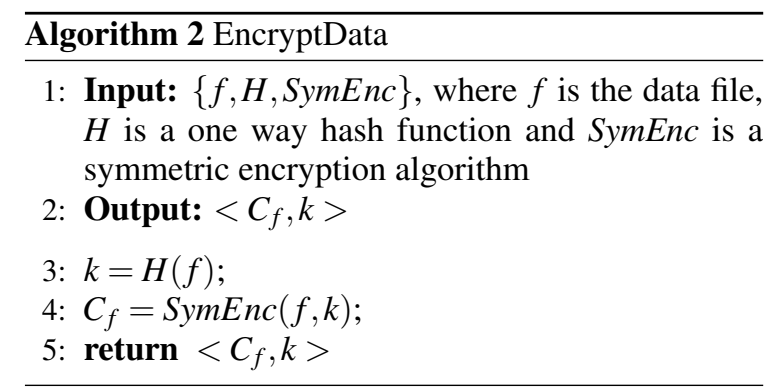

is, CloudaSec introduces a novel asymmetric key encoding, to ensure flexible sharing of outsourced data. For instance, the resulting enciphered key involves a couple of elements $\left\langle C_{1}, C_{2}\right\rangle$. $C_{1}$ is included in the user metadata, by the depositor $U_{i}$. However, $C_{2}$ is sent to the CSP, in order to grant additional access verifications on the outsourced data and to generate a redirected access key element. We assume in our ap-

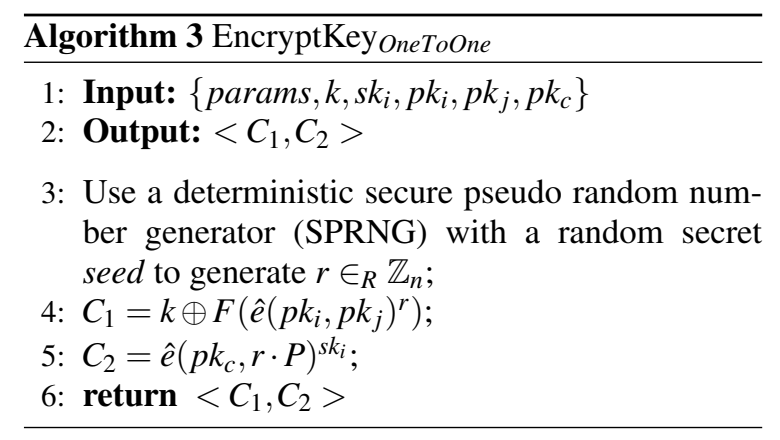

proach that all key elements belong to a finite domain space $D$. We denote each key element by key.elt as defined in Equation 1, where $D$ can be either a user metadata element space or a CSP metadata element space.

$$
\text { key.elt } t_{i \in\{1,2,3\}}=\left\{C_{i}, D\left(C_{i}\right)\right\}_{i \in\{1,2,3\}}
$$

We must note that the CSP has a pair of private and public keys as $\left\langle s k_{c}, p k_{c}\right\rangle$, where $s k_{c}=s_{c} \in_{R} \mathbb{Z}_{n}$ presents the provider private key and $p k_{c}=s_{c} \cdot P \in \mathbb{G}_{1}^{*}$ is his related public key. When the CSP receives the second key element $C_{2}$, he runs the ShrinKey algorithm in order to derive a redirected key element $C_{3}$, as presented in Algorithm 4. This latter enciphers $C_{2}$, using his secret key $s k_{c}$ and generates the corresponding $C_{3}$. Afterwards, when the CloudaSec recipient $U_{j}$, where $j \neq i$, wants to recover the outsourced data file, he has to retrieve the encrypted data key $\left\langle C_{1}, C_{3}\right\rangle$. As such, the recipient user $U_{j}$ starts a data backup scenario as follows.

After successfully authenticating with the CSP, $U_{j}$ gets the redirected key element $C_{3}$. Then, based on the outsourced user metadata, the authorized recipi- 


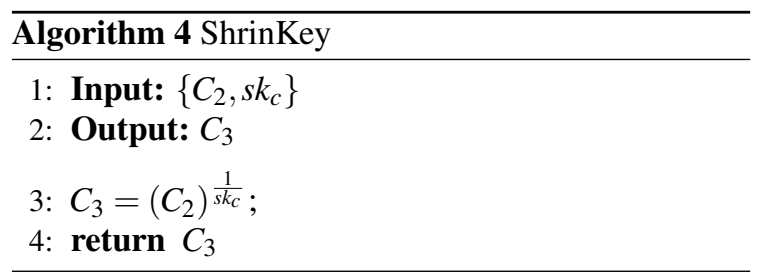

ent $U_{j}$ extracts the $C_{1}$ key element, which was enciphered, using his public key $p k_{j}$ by the depositor $U_{i}$. In the sequel, based on his local secret key $s k_{j}$, the recipient $U_{j}$ performs the DecryptKey OneToOne procedure, in order to decipher the encrypted data key $k$, as presented in Algorithm 5. Finally, the recipient

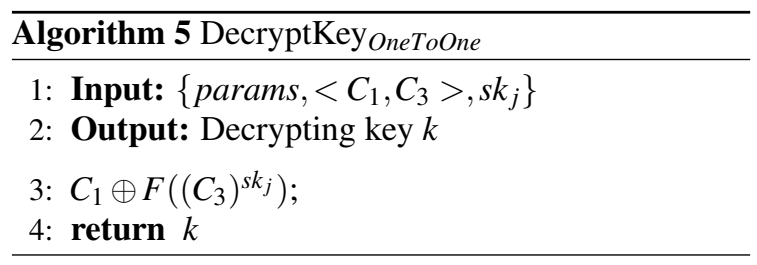

retrieves the data file content. That is, he locally runs the CloudaSec DecryptData procedure, based on the derived deciphering key $k$, using a symmetric algorithm over encrypted data $C_{f}$ (cf. Algorithm 6).

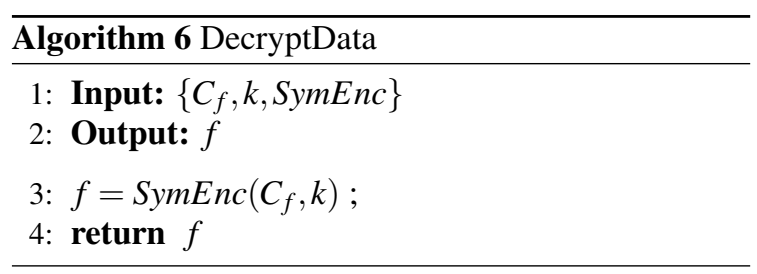

\subsubsection{CloudaSec One to Many Sharing Scenario}

When a depositor $U_{i}$ intends to share data with a multi-user group, he has to encipher the data decrypting key based on his public key $p k_{i}$ and a secret shared group key $d$. The secret shared key is a private key, only known to the authorized group members. It is derived by performing the key agreement algorithm (Algorithm ??), based on parallel Diffie Hellman instantiations (Burmester and Desmedt, 2005), as explained in Section 3.5.

The depositor executes the EncryptKeyOneToMany procedure (cf. Algorithm 7), in order to encrypt the deciphering data key. The resulting encrypted key includes a couple of elements $\left\langle C_{1}, C_{2}\right\rangle$, where $C_{1}$ is integrated in user metadata by the depositor, and $C_{2}$ is sent to the cloud provider, in order to generate an accessing key $C_{3}$ element (Algorithm 4). When an au-

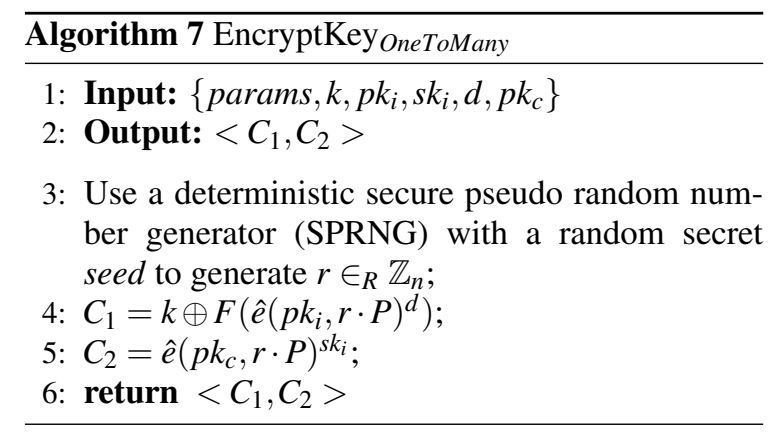

thorized group member wants to retrieve the data decrypting key, he has first to send a request to the cloud provider to access to the outsourced data. The CSP verifies the granted privileges of the requesting user. Once accepted, the requesting group member receives the redirected key element $C_{3}$ obtained by performing the ShrinKey procedure, as shown in Section 3.4.1. Then, he runs the CloudaSec DecryptKeyOneToMany procedure (cf. Algorithm 8) using the secret shared group key $d$, in order to derive the deciphering data key $k$.

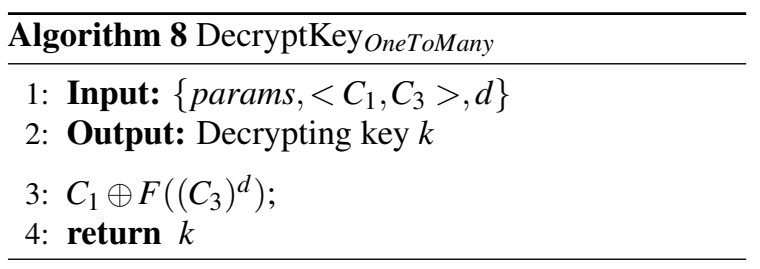

\subsection{CloudaSec Management Layer Procedures}

Efficient data sharing between authorized cloud users, among dynamic groups remains a challenging concern. That is, it increases the computation complexity and the bandwidth consumption, due to the sharing of group secret keys. In addition, the heavy overhead and the large size of outsourced data may reduce the advantages of remote sharing services to resourceconstrained devices.

In order to tackle this challenging issue, CloudaSec introduces the role of a group manager (GM). This latter is responsible for elementary procedures, namely the initialization of the group parameters and the organization among authorized registered group members. Then, the GM makes the group parameters available by migrating them to the cloud. Such a design can significantly reduce the computation overhead, at the CloudaSec user side.

Let us consider $G r=\left\{\left\{U_{0}, i d_{0}\right\}, \ldots,\left\{U_{N-1}, i d_{N-1}\right\}\right\}$ a dynamic group of $N$ users. These group members 
want to generate a common secret $d \in \mathbb{Z}_{n}$. In the following, we denote by pubelts $s_{i}$ the public elements of a CloudaSec registered group member $U_{i}$ as described in Equation 2.

$$
\text { pubelt }_{i}=<i d_{i}, p k_{i}>
$$

where $i \in\{1, \ldots, N-1\}$ and $N$ is the number of group users including the manager. As such, we note that the couple $<i d_{0}, p k_{0}>$ presents the public group elements of the Group Manager (GM). First, the GM

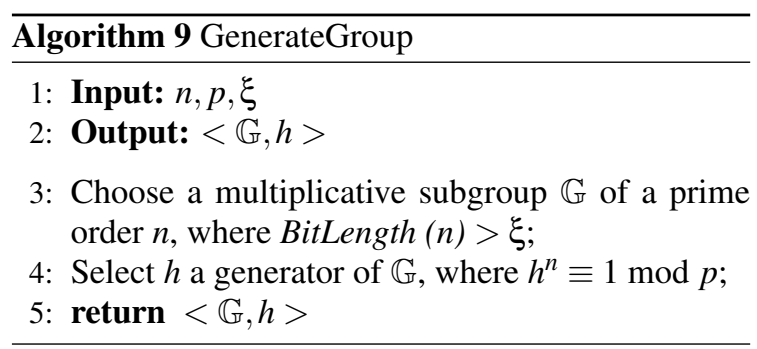

runs a GenerateGroup procedure, in order to derive a multiplicative group and makes public the output of this algorithm, which is used to generate the secret group key $d$ (cf. Algorithm 9). We must note that the order of the multiplicative group is strongly associated to the security level $\xi$ of the cryptographic algorithms.

Then, with respect to the published multiplicative group $\mathbb{G}$, each group user $U_{i}$ chooses a random $b_{i}$ and locally runs the CloudaSec UserKeyShareElt procedure in order to get his first key share element $h_{i}$, as presented in Algorithm 10.

The GM receives the public elements pubelt $s_{i}$ of each

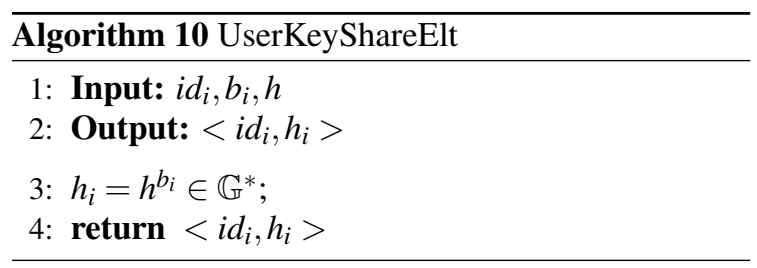

group member $U_{i}$. Then, he updates a list of non revoked users $L_{N R}$, which contains the public elements of all non revoked group users. This list sets the authorized group members arranged into a cycle. As such, each user can easily identify his predecessor $U_{i-1}$ and his successor $U_{i+1}$. Thus, using the CloudaSec GenerateGroup and UserKeyShareElt procedures, $U_{i}$ computes his group key share $\left(h_{i}, X_{i}\right)$, as depicted in Equation 3.

$$
\left(h_{i}, X_{i}\right)=\left(h^{b_{i}},\left(\frac{h_{i+1}}{h_{i-1}}\right)^{b_{i}}\right)
$$

Once computed, each user $U_{i}$ sends his group key share to the GM. This latter publishes the received key shares of the non revoked users, as presented in Table 2. Then, based on Algorithm ??, each user should derive the secret group key $d$, using the published elements in the $L_{N R}$ list, while respecting the ring construction of the group members (Burmester and Desmedt, 2005).

Table 2: List of Non Revoked users $L_{N R}$

\begin{tabular}{|c|c|c|}
\hline Group id & User Pubelts & User Key Share \\
\hline \multirow{3}{*}{$i d_{G r}$} & $U_{0}\left(i d_{0}, p k_{0}\right)$ & $\left(h_{0}, X_{0}\right)$ \\
\cline { 2 - 3 } & $U_{1}\left(i d_{1}, p k_{1}\right)$ & $\left(h_{1}, X_{1}\right)$ \\
\cline { 2 - 3 } & $\vdots$ & $\vdots$ \\
\cline { 2 - 3 } & $U_{N-1}\left(i d_{N-1}, p k_{N-1}\right)$ & $\left(h_{N-1}, X_{N-1}\right)$ \\
\hline
\end{tabular}

\subsubsection{User Subscription}

When a new user $\left\{U_{N}, i d_{N}\right\}$ wants to join the group $G r$, presented by $G r=$ $\left\{\left\{U_{0}, i d_{0}\right\}, \ldots,\left\{U_{N-1}, i d_{N-1}\right\}\right\}, \quad$ where $i \notin$ $\{0, \ldots, N-1\}$, he first runs the UserKeyShareElt algorithm in order to get his public key share element $\left.<i d_{N}, h_{N}\right\rangle$. Then, the new group member computes and sends his key share $\left(h_{N}, X_{N}\right)$ to the group manager. Hence, The GM sends a notification message to the remaining group members and updates the list of non revoked users $L_{N R}$.

Afterwards, each group user computes the new secret key $d_{N}$, due to the group state modification. Since the derivation of the group secret key depends on members' identifiers, the computation of key shares $\left(h_{i}, X_{i}\right)$ may be restricted to the solicited members. Consequently, CloudaSec significantly saves the processing time and storage cost at CloudaSec user side.

The user subscription operation prevents new users from accessing to protected content, before joining the group. As such, CloudaSec ensures the forward security. In order to grant access privileges to new subscribers to outsourced data, the sharing of a secrets' list $L_{S}$ is required.

Indeed, the group manager updates a list of previously used secrets $L_{S}$ by including the new group secret key. Then, he sends it to the CSP in an encrypted format by using symmetric encryption algorithm and the derived secret group key $d$. In the sequel, any authorized group member authenticates with the CSP and uploads the encrypted list. So that, he can obtain $L_{S}$ using the derived secret group key and the symmetric decryption algorithm. 


\subsubsection{User Revocation}

When a group member $U_{j}$ leaves or is revoked from the group $G r=\left\{i d_{0}, i d_{1}, i d_{2}, \ldots, i d_{k}\right\}$, where $j \in$ $\{0,1, \ldots, k\}$, the group manager first updates the list of non revoked users $L_{N R}$. That is, he removes the public elements $\left\langle i d_{j}, p k_{j}>\right.$ and the key share $\left(h_{N}, X_{N}\right)$ of the revoked member from the $L_{N R}$ list. Then, he sends a notification message to other group users and sends the updated list to the CSP. Each group user computes a new secret key $d_{N}$ by running the GroupKey algorithm.

We note that the number of the revoked users $R U$ has to be strictly less than $(N-1)$, in order to keep the One To Many sharing scenario. In fact, we consider two cases.

1. Case 1 - There are $R U$ revoked users, where $1 \leq R U \leq N-2$. The group manager revokes $R U$ users, and updates the $L_{N R}$ list. That is, he withdraws the revoked users' identities and reorganizes the indexing system of the list. The group manager optimizes the changes of the group list based on a selection protocol, in order to save the computation capacities of resource constrained devices. As such, a non solicited group member is requested to only compute the resulting group key, using the published public group elements.

2. Case 2 - There are $R U$ revoked users, where $R U \geq N-1$. In this case, the group manager is released from his role. As such, the multi-user group becomes a duo group that shares data based on a sharing One To One scenario.

\section{SECURITY ANALYSIS}

In the following security analysis, we discuss the resistance of CloudaSec against two adversaries, based on a realistic threat model. We briefly present the security of our proposed framework in terms of access control and data file confidentiality.

\subsection{Threat Model}

For designing the most suitable security solutions for cloud storage, we have to consider realistic threat models. That is, we point out two adversaries: malicious cloud user and honest but curious cloud server.

- malicious user adversary - an attacker can be either a revoked user with valid data decryption keys, an unauthorized group member or a group member with limited access rights. As such, he targets to get access to the outsourced shared data.
The objective of a malicious user is to convince the cloud server that he is a legitimate data owner. That is, we suppose that the adversary successes to gain knowledge of an arbitrary part of the decrypting key.

- curious cloud server adversary - this storage server honestly performs the operations defined by our proposed scheme, but it may actively attempt to gain the knowledge of the outsourced sensitive data.

CloudaSec must provide the capabilities to the clients and the service provider to thwart the two threats mentioned above. To this end, our proposed framework must enforce a mutual verification of the actions conducted by a CloudaSec client and the storage server.

\subsection{Data Confidentiality}

In our model, data files are stored encrypted in cloud servers using a symmetric encryption algorithm, and the secret key is protected relying on an asymmetric scheme, in order to ensure efficient access control. As such, the data confidentiality preservation is tightly related to security of the used symmetric algorithm and the secrecy of the data key.

Theorem 4.1. data confidentiality preservation The proposed framework supports data confidentiality preservation.

Proof. The confidentiality of data contents is twofold. First, it depends on the security level of the encryption algorithm. This latter is a recurrent concept in cryptography. It permits to evaluate the hardness of breaking an encryption or a signature algorithm. That is, the harder the level of security is, the harder the cryptanalysis of the algorithm becomes. Our employed encryption algorithm inherits the unforgeable property from the selected scheme. Therefore, CloudaSec ensures the confidentiality of encrypted content exposed in public cloud servers.

Second, the confidentiality of data relies also on the secrecy of the deciphering key hosted in cloud servers. The demonstration of this state is derived from these two lemmas.

Lemma 4.2. Unauthorized users cannot decrypt the deciphering data keys.

Proof. The proof of this lemma is equivalent to the security of the key encryption algorithms and the correctness of the key decryption algorithms.

Let us suppose that an unauthorized user can be a revoked group member or a malicious cloud user. Thus, a brief security analysis can be done on the three following cases. 
- Case $A$ - a revoked group member $U_{R}$ should not be able to decrypt new data contents, using the old group secret key $d$. This latter knows the public elements of the non revoked users published in $L_{N R}$ and the previous organization of the group arranged into a cycle. Moreover, he can merely guess the solicited members after his revocation. As such, taking advantage from published information, $U_{R}$ tries to deduce the new group secret key $d_{N}$ or to extract a data key after his revocation from the group. In this case, we may consider two different sessions $(\alpha)$ and $(\beta)$, where the same data owner $U_{i}$ shares two different data files $f_{\alpha}$ and $f_{\beta}$, after the revocation of $U_{R}$. In the sequel, two key elements are defined as follows:

$$
\begin{gathered}
C_{1}^{(\alpha)}=k_{\alpha} \oplus F\left(\hat{e}\left(p k_{i}, r_{\alpha} \cdot P\right)^{d_{N}}\right) \\
C_{1}^{(\beta)}=k_{\beta} \oplus F\left(\hat{e}\left(p k_{i}, r_{\beta} \cdot P\right)^{d_{N}}\right)
\end{gathered}
$$

On one side, knowing the public key of the depositor $p k_{i}$, we state that the deduction of the new group secret $d_{N}$ from $C_{1}^{(\alpha)}$ cannot hold. Obviously, this is due to the usage of a random value $r_{\alpha}$. We also state that our scheme inherits the unforgeablility property from the Burmester key distribution algorithm (Burmester and Desmedt, 2005). On the other side, $U_{R}$ cannot deduce secret information from $C_{1}^{(\alpha)} \oplus C_{1}^{(\beta)}$, mainly due to the exclusive-or function.

As such, a revoked group member $U_{R}$ has no advantage to guess the new secret group, based on the old group key $d$ and the previously published public elements. However, we must note that CloudaSec does not prevent a revoked member from decrypting previously shared contents.

- Case B - The main advantage of a malicious cloud user is to deduce information from an unbounded number of sessions, where the same data owner shares different contents with legitimate group members. As such, two different cases are exposed as follows.

On one hand, in a one to many sharing scenario, let us suppose that a user $U_{i}$ shares two data files $f_{1}$ and $f_{2}$, respectively enciphered based on two different keys $k_{1}$ and $k_{2}$, using the same random $r$. That is, based on Equation 4 and Equation 5, an attacker obtains indistinguishable data key elements key.elt 1 , as follows.

$$
\begin{aligned}
& C_{1}^{\left(f_{1}\right)}=k_{1} \oplus F\left(\hat{e}\left(p k_{i}, r \cdot P\right)^{d}\right) \\
& C_{1}^{\left(f_{2}\right)}=k_{2} \oplus F\left(\hat{e}\left(p k_{i}, r \cdot P\right)^{d}\right)
\end{aligned}
$$

Hence, we notice that there is no polynomial-time algorithm that can deduce secret information from
Equation $4 \oplus$ Equation $5=k_{1} \oplus k_{2}$, due to the usage of the exclusive-or function. As such, the secrecy disclosure of the deciphering keys remains infeasible.

On the other hand, in a one to one sharing scenario, we suppose that a depositor $U_{i}$ shares data with one recipient $U_{j}$, using the same random secret $r$, in order to encipher different data decrypting key. Thus, based on two successive sessions $(\alpha)$ and $(\beta)$, the malicious cloud user gets the following key elements key.elt $t_{1}$.

$$
\begin{aligned}
& C_{1}^{(\alpha)}=k_{1} \oplus F\left(\hat{e}\left(p k_{i}, p k_{j}\right)^{r^{(\alpha)}}\right) \\
& C_{1}^{(\beta)}=k_{2} \oplus F\left(\hat{e}\left(p k_{i}, p k_{j}\right)^{r^{(\beta)}}\right)
\end{aligned}
$$

Therefore, the deduction from the enciphered contents is protected, due to the usage of different data encryption keys. In addition, the security of metadata takes advantages from the properties of the exclusive-or function which ensure the indistinguishability of encryptions.

- Case $C$ - Let us suppose that a depositor $U_{i}$ belongs to two different groups $G_{A}$ and $G_{B} . U_{i}$ wants to share the same data file $f$ to these two groups. $G_{A}$ and $G_{B}$ have two secrets $d_{A}$ and $d_{B}$, respectively. As such, we have two different key elements key.elt $t_{1}$, as follows:

$$
\begin{aligned}
& C_{1}^{(A)}=k \oplus F\left(\hat{e}\left(p k_{i}, r_{A} \cdot P\right)^{d_{A}}\right) \\
& C_{1}^{(B)}=k \oplus F\left(\hat{e}\left(p k_{i}, r_{B} \cdot P\right)^{d_{B}}\right)
\end{aligned}
$$

We suppose that there a malicious group member $U_{M}$ that belongs to the group of users $G_{A} . U_{M}$ tries to deduce the group secret key $d_{B}$ of $G_{B}$.

From Equation 6, the recipient $U_{M}$ extracts the data deciphering key $k$. In the sequel, from $C_{1}^{(B)}$, $U_{M}$ computes Equation 8 as follows:

$$
\begin{aligned}
C_{1}^{(B)} \oplus k & =k \oplus F\left(\hat{e}\left(p k_{i}, r_{B} \cdot P\right)^{d_{B}}\right) \oplus k \\
& =F\left(\hat{e}\left(p k_{i}, r_{B} \cdot P\right)^{d_{B}}\right)
\end{aligned}
$$

From Equation 7 and Equation 8, the malicious recipient $U_{M}$ calculates $\hat{e}\left(p k_{i}, r_{A} \cdot P\right)^{d_{A}} \star$ $\left[1 /\left(\hat{e}\left(p k_{i}, r_{B} \cdot P\right)^{d_{B}}\right)\right]$. So that, $U_{M}$ executes the following steps:

$$
\hat{e}\left(s k_{i} \cdot P, r_{A} \cdot P\right)^{d_{A}} \star\left[\frac{1}{\hat{e}\left(s k_{i} \cdot P, r_{B} \cdot P\right)^{d_{B}}}\right]=\frac{g^{r_{A} d_{A}}}{g^{r_{B} d_{B}}}
$$

Knowing the group secret $d_{A}$ and the two quantities $g^{r_{A} d_{A}}$ and $g^{r_{B} d_{B}}, U_{M}$ cannot extract the group secret $d_{B}$. Obviously, this contradicts the $\mathrm{CDH}$ assumption.

Finally, as data may be shared by different depositors or groups, and these depositors may belong to several groups, our framework strongly ensures the confidentiality of outsourced contents, based on the hardness of the $\mathrm{CDH}$ assumption. 
Lemma 4.3. The CSP is unable to learn the content of outsourced data files in his public servers, based on the CDH assumption.

Proof. A curious CSP tries to access to the stored data contents. His main problem is that outsourced data files are encrypted. However, the CSP tries to gain knowledge of an arbitrary part of secret information. That is, after each storage of data content, the CSP receives the second key element key.elt $t_{2}$, from the depositor $U_{i}$ as $C_{2}=\hat{e}\left(p k_{c}, r \cdot P\right)^{s k_{i}}$, where $s k_{i} \in \mathbb{Z}_{n}$ is the secret element of the depositor $U_{i}$ and $p k_{c}=s k_{c} \cdot P \in$ $\mathbb{G}_{1}^{*}$ is the public key of the storage provider. As such, in order to extract secret information, the CSP computes $\hat{e}\left(p k_{i}, P\right)=g^{s k_{i}}$. This deduction cannot hold. Clearly, this contradicts the $\mathrm{CDH}$ assumption.

Given the redirected key element $C_{3}=C_{2}^{\frac{1}{c}}=$ $\left(\hat{e}\left(p k_{c}, r \cdot P\right)^{s k_{i}}\right)^{\frac{1}{c}}$, the CSP computes $C_{3}=\left(\hat{e}\left(p k_{i}, r\right.\right.$. $\left.P)^{s k_{c}}\right)^{\frac{1}{c}}=\hat{e}\left(p k_{i}, r \cdot P\right)$. Then, he executes Equation 10 and Equation 11 as follows:

$$
\begin{aligned}
& C_{2} \star\left[\frac{1}{\hat{e}\left(p k_{c}, p k_{i}\right)}\right]=\frac{g^{s k_{i} s k_{c} r}}{g^{s k_{i} s k_{c}}}=g^{r} \\
& C_{3} \star\left[\frac{1}{\hat{e}\left(p k_{i}, p k_{i}\right)}\right]=\frac{g^{s k_{i} r}}{g^{s k_{i}^{2}}}=\frac{g^{r}}{g^{s k_{i}}}
\end{aligned}
$$

From Equation 10 and Equation 11, this curious storage server cannot extract the secret key of the depositor $s k_{i}$ or the used random value $r$. As such, the storage server cannot learn the content of the outsourced data files, based on the hardness of the $\mathrm{CDH}$ assumption.

\subsection{Access Control}

CloudaSec is designed to ensure forward and backward secrecy. When a new user joins the group or a group member is revoked, a notification message is sent to CSP and to the remaining members, in order to adjust the access control lists.

On one side, when a new user $U_{N}$ joins the group, he has to generate his own group public elements pubelt $_{N}$. These elements will be later used to derive the new group key $d_{N}$. On the other side, when a user $U_{R}$ leaves the group, the GM updates the sharing lists, in order to generate the new decrypting key. Consequently, a new user cannot decrypt the old data files, using the new derived key, and a revoked user cannot decrypt new files, with the old deciphering key.

The access control preservation is ensured, based on the following two lemmas.
Lemma 4.4. Key Decryption Correctness Unrevoked users are able to access the cloud.

Proof. The proof of this lemma is equivalent to the correctness of the key decryption algorithms, on the basis of the two sharing scenarios, as follows.

- One To One sharing scenario - the decryption holds if, and only of the decrypting key $k^{*}=$ $C_{1} \oplus F\left(\left(C_{3}\right)^{s k_{j}}\right)$.

This verification holds as follows. On one side, the authorized recipient $U_{j}$ computes the data key element included in user metadata as follows:

$$
\begin{aligned}
C_{1} & =k \oplus F\left(\hat{e}\left(p k_{i}, p k_{j}\right)^{r}\right) \\
& =k \oplus F\left(\hat{e}\left(s k_{i} \cdot P, s k_{j} \cdot P\right)^{r}\right) \\
& =k \oplus F\left(\hat{e}\left(s_{i} \cdot P, s_{j} \cdot P\right)^{r}\right)
\end{aligned}
$$

On the other side, the cloud provider sends the redirected key element $C_{3}$ to the CloudaSec recipient, which is computed as follows.

$$
\begin{aligned}
C_{3} & =\left(C_{2}\right)^{\frac{1}{s_{c}}} \\
& =\left(\hat{e}\left(p k_{c}, r \cdot P\right)^{s k_{i}}\right)^{\frac{1}{s_{c}}} \\
& =\left(\hat{e}\left(s k_{c} \cdot P, r \cdot P\right)^{s_{i}}\right)^{\frac{1}{s_{c}}} \\
& =\left(\hat{e}\left(s_{c} \frac{1}{s_{c}} \cdot P, r \cdot P\right)^{s_{i}}\right) \\
& =\left(\hat{e}(P, r \cdot P)^{s_{i}}\right)
\end{aligned}
$$

In the sequel, given the non singularity property of the bilinear functions, the verification holds if, and only if $k^{*}=C_{1} \oplus F\left(\left(C_{3}\right)^{s k_{j}}\right)$, where $C_{1} \oplus$ $F\left(\left(C_{3}\right)^{s k_{j}}\right)$ is denoted by $(E)$.

$$
\begin{aligned}
(E) & =k \oplus F\left(\hat{e}\left(s k_{i} \cdot P, s k_{j} \cdot P\right)^{r}\right) \oplus F\left(\hat{e}(P, r \cdot P)^{s k_{i} s k_{j}}\right) \\
& =k \oplus F\left(\hat{e}\left(s_{i} \cdot P, s_{j} \cdot P\right)^{r}\right) \oplus F\left(\hat{e}\left(s_{i} \cdot P, r \cdot P\right)^{s_{j}}\right) \\
& =k \oplus F\left(\hat{e}\left(s_{i} \cdot P, s_{j} \cdot P\right)^{r}\right) \oplus F\left(\hat{e}\left(s_{i} \cdot P, s_{j} \cdot P\right)^{r}\right) \\
& =k
\end{aligned}
$$

- One To Many sharing scenario - the decryption holds if, and only of the data key $k^{*}=C_{1} \oplus$ $F\left(\left(C_{3}\right)^{d}\right)$. This verification holds as follows.

On one hand, the authorized group member $U_{j}$ computes the data key element $C_{1}$ included in user metadata as follows:

$$
\begin{aligned}
C_{1} & =k \oplus F\left(\hat{e}\left(p k_{i}, r \cdot P\right)^{d}\right) \\
& =k \oplus F\left(\hat{e}\left(s k_{i} \cdot P, r \cdot P\right)^{d}\right)
\end{aligned}
$$

On the other hand, the CSP executes the following operations on key.elt 2 , in order to get the redi- 
rected element $C_{3}$

$$
\begin{aligned}
C_{3} & =\left(C_{2}\right)^{\frac{1}{s_{c}}} \\
& =\left(\hat{e}\left(p k_{c}, r \cdot P\right)^{s k_{i}}\right)^{\frac{1}{s_{c}}} \\
& =\left(\hat{e}\left(s k_{c} \cdot P, r \cdot P\right)^{s_{i}}\right)^{\frac{1}{s_{c}}} \\
& =\left(\hat{e}\left(s_{c} \frac{1}{s_{c}} \cdot P, r \cdot P\right)^{s_{i}}\right) \\
& =\left(\hat{e}(P, r \cdot P)^{s_{i}}\right)
\end{aligned}
$$

As presented in the One To One sharing scenario, given the non singularity property of the bilinear functions, the verification holds if, and only if $k^{*}=C_{1} \oplus F\left(\left(C_{3}\right)^{d}\right)$, where $C_{1} \oplus F\left(\left(C_{3}\right)^{d}\right)$ is denoted by $(F)$.

$$
\begin{aligned}
(F) & =k \oplus F\left(\hat{e}\left(s k_{i} \cdot P, r \cdot P\right)^{d}\right) \oplus F\left(\hat{e}(P, r \cdot P)^{s k_{i} d}\right) \\
& =k \oplus F\left(\hat{e}\left(s_{i} \cdot P, r \cdot P\right)^{d}\right) \oplus F\left(\hat{e}\left(s_{i} \cdot P, r \cdot P\right)^{d}\right) \\
& =k
\end{aligned}
$$

We state that the authorized CloudaSec users are able to decipher the decrypting data key, thanks to the correctness of the key decryption algorithms.

Lemma 4.5. Unauthorized entities are unable to access the cloud.

Proof. The proof of this lemma is twofold.

On one side, after each group member $U_{R}$ revocation, the group manager updates the list $L_{N R}$ and sends a notification message to the authorized registered group members. Then, he communicates this list to the cloud provider. This latter sends $L_{N R}$ to the remaining group members after a mutual authentication, in order to verify the updated organization of the group.Then, the remaining group members compute the new secret group key $d_{N}$ by performing the GroupKey algorithm. Therefore, the new data keys are encrypted by using EncryptKeyOneToMany algorithm.

As discussed in Lemma 4.2 and Lemma 4.4, only authorized recipients, knowing the new key $d_{N}$, are able to decrypt the enciphered data. However, the CSP and the revoked users cannot extract the key $d_{N}$, based on the previously published public elements and the list of authorized members $L_{N R}$. This is mainly due to the computation of the group secret which requires the private secret key $s k_{i}$ of each deriving group member $U_{i}$.

On the other side, when a group user wants to access the cloud, the CSP has to verify the access control list. That is, the cloud provider gives or rejects access to data contents, based on the granted privileges of the requesting recipient.

\section{PERFORMANCE EVALUATION}

In this section, we first present the context of CloudaSec implementation with OpenStack Object Storage, and then evaluate the system performances, in terms of computation, communication and storage costs.

\subsection{Context}

In order to evaluate the performances of our proposal, we build a simulated CloudaSec framework, based on OpenStack Storage system (Swift) (swi, ). Swift is a cloud based storage system, which stores data and allows write, read, and delete operations on them. To achieve security enhancement of Swift, we extend its functionalities with algorithms and protocols designed in CloudaSec.

We have designed a simplified CloudaSec architecture, based on Swift. Indeed, our architecture consists in dividing the machine drive into four physical volumes. Then, each volume is divided into four logical volumes. In total, we obtain sixteen partitions, each one represents one logical storage zone.

The simulation consists of two components: the client side and the cloud side. We implement data layer cryptographic algorithms based on cryptographic functions from the Open-SSL library (The OpenSSL Project, 2003), the GMP library (et al., 2002) and the Pairing Based Cryptography (PBC) library (Ben, 2007), with independent native processes. We choose Advanced Encryption Standard (AES) as our symmetric encryption algorithm and implement the $C B C$ mode of AES.

We have conducted a number of experiments to evaluate CloudaSec in the system and cloud levels. We study the client efficiency of the cryptographic algorithms with different pairing types and the user management costs for communication and storage.

\subsection{Computation Cost Evaluation}

In order to evaluate the performances at the client side, we conduct data encryption and decryption tests locally. For our tests, we used 1000 samples in order to get our average durations. In addition, we conducted our experiments on an Intel core 2 duo, started on single mode, where each core relies on $800 \mathrm{MHz}$ clock frequency (CPU).

Figure 2 shows the computation overhead of data encryption and decryption at the client side, with different sizes of data contents. We can notice that the data encryption takes less than $12 \mathrm{~ms}$, in order to encrypt $1 M B$ data file. We can note that this computa- 

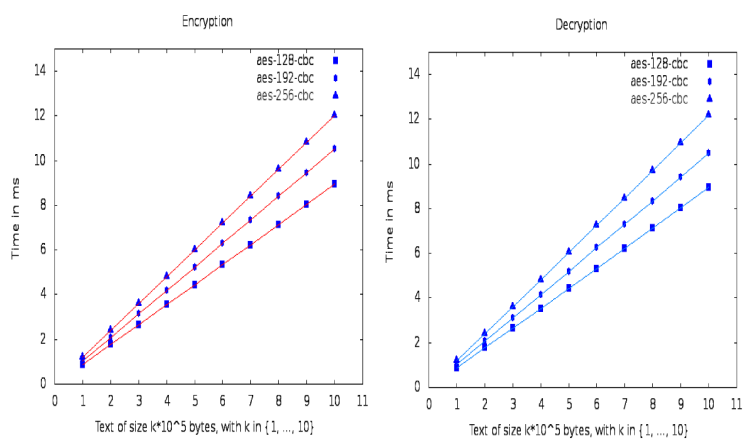

Figure 2: Computation overhead of data encryption and decryption at the client side with different data size (from $10^{5}$ to $10^{6}$ bytes) (ms)

tion cost remains attractive, as it provides better security to outsourced data and does not deserve the client resources.

Then, we perform the encryption of the deciphering data key $k$. That is, as our proposed framework relies on the use of bilinear maps, we choose two symmetric pairing functions from the PBC library (Ben, 2007), including type E pairing e.param and type A a.param. Thus, we examine the impact of different bilinear functions on the performances of CloudaSec, while considering three different security levels (cf. Figure 3).

In cryptography, the security level of a symmetric encryption algorithm is defined as the number of operations needed to break the algorithm when a $k$-bit key length is used. The security level in our proposal depends on the security level of the bilinear function in use $\hat{e}$, which is related to the hardness of solving the ECDLP in $\mathbb{G}_{1}$. As such, it is closely related to the groups being selected. As shown in Figure 3 , the encryption time increases along with the security level, while there is a tiny difference between the two symmetric pairing functions. As such, the type of the pairing function should be taken into account, while implementing CloudaSec data layer procedures. We must note that the type of the pairing function is bound to the choice of the elliptic curve, where the bilinear map is computed.

Finally, we investigate the impact of the cryptographic operations, at CloudaSec client side. We compare the encryption duration against OpenStack upload and download duration, as depicted in Figure 4. In fact, we examine the encryption operations vs Swift upload procedure and the decryption operations vs Swift download procedure. We must note that the computation times include the key generation and the data encryption with $A E S-256-C B C$ mode. We notice that the cryptographic operations, at the client side are acceptable compared to the upload operations

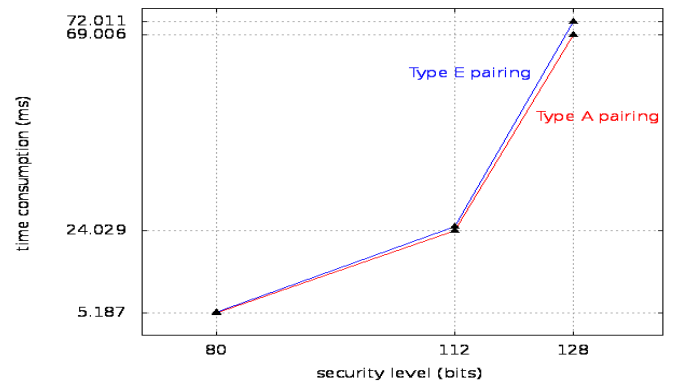

Figure 3: Computation duration of Type A vs Type E pairing functions (ms)

and do not carry exhaustive computation capacities. For example, a $8 * 10^{5}$ bytes data size requires only 0.1 seconds to be enciphered, compared to $10 \mathrm{sec}$ ond be uploaded. Therefore, the encryption procedures involve $1 \%$ from the OpenStack upload overhead. As such, CloudaSec does not deserve the client resources, and presents an interesting processing cost for resource constrained devices.
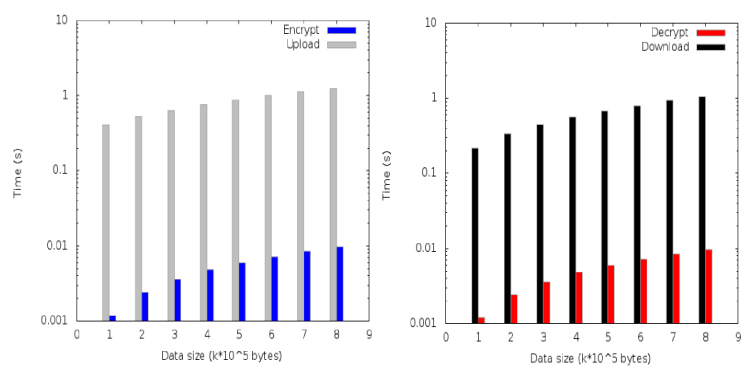

Figure 4: Impact of cryptographic operations on CloudaSec at the client side $\left(\log _{10}(m s)\right)$

\subsection{Communication Cost Evaluation}

We investigate the communication overhead, when a client stores his data file to remote servers and then when he retrieves the outsourced content. As such, we conduct some experiments with different data sizes and we evaluate the upload and download times of encrypted contents, as shown in Figure 5.

We can notice that the average communication times are merely stable, with small data sizes, for the storage and backup scenarios. However, this overhead gradually increases, when the client intends to store large data contents. We also analyze the communication cost, due to a group update, namely when a new user wants to join the group. In our tests, we are based on pre-computed tables, in order to optimize the computation cost to resource-constrained devices. Thus, we consider that the group includes 10 members at the beginning. Then, 10 users join the group simulta- 

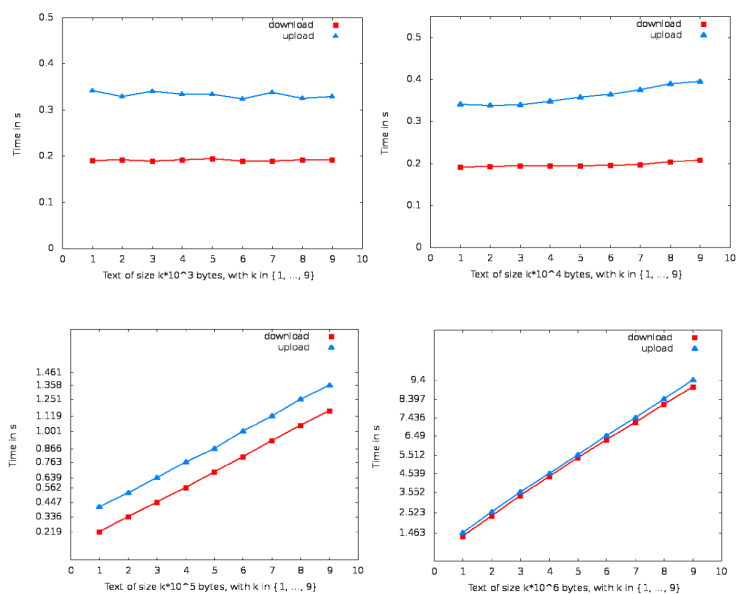

Figure 5: OpenStack upload and download overhead with different data size (ms)

neously, until reaching 100 members. We recall that the computation complexity of the group update increases with respect to the number of new subscribers, as presented in Section 3.3.

As depicted in Figure 6, the derivation of the new secret group key takes less than 6 ms for 100 users. This computation cost remains interestingly attractive, along with our broadcasting approach.

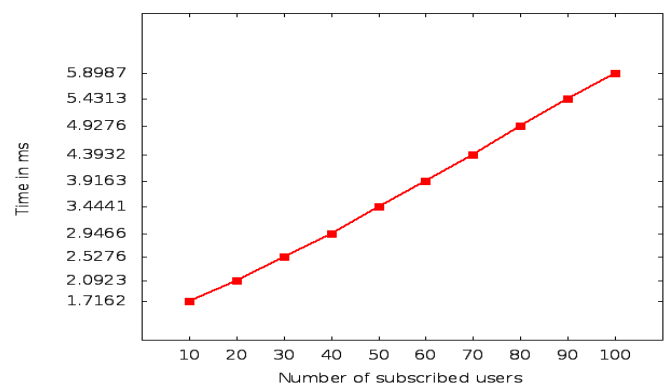

Figure 6: Computation complexity of a group update (ms)

\subsection{Storage Cost Evaluation}

We investigate the storage cost for the key management operations at the client side. In order to maintain a group membership, a registered user has only to keep the secret group key.

Let suppose that the security parameter $\xi=80$ bits. We denoted by $E\left(\mathbb{F}_{n}\right)$ the elliptic curve defined over the finite prime field $\mathbb{F}_{n}$. Meanwhile, we denote $\hat{e}: \mathbb{G}_{1} \times \mathbb{G}_{1} \longrightarrow \mathbb{G}_{2}$ the bilinear function. $\mathbb{G}_{1}$ corresponds to the q-torsion subgroups of $E\left(\mathbb{F}_{n}\right)$ and $E\left(\mathbb{F}_{n^{k}}\right)$ where $k$ is the embedding degree of the curve $E . \mathbb{G}_{2}$ is a multiplicative subgroup of $\mathbb{F}_{n^{k}}$ of order $q$. For example, according to the security parameter $\xi=$
80 , we set $q=160$ bits and $n=512$ bits length, while the embedding degree is equal to 2 . As such, $\mathbb{G}_{2}$ is a subgroup of $\mathbb{F}_{n^{2}}$ which has a order 1024 bits order. Therefore, a client has to locally keep a secret $d$, where $|d|=160$. As such, CloudaSec introduces an attractive storage cost, especially for limited storage capacities.

\section{RELATED WORK}

Several security solutions have been recently developed, in order to provide data confidentiality in public cloud storage environments \{ (Xiong et al., 2012),(Zarandioon et al., 2011), (Yu et al., 2010), (Zhou et al., 2011), (Liu et al., 2013),(Fugkeaw, 2012)\}, while considering the group access control issues.

In (Yu et al., 2010), Yu et al. proposed an attribute based access control policy to securely outsource sensitive client data to untrusted cloud servers. In this approach, data are encrypted using a symmetric encryption algorithm, while the enciphering key is protected by a Key-Policy-Attribute Based Encryption scheme (Goyal et al., 2006). In order to manage dynamic groups, they delegate the key re-encryption procedures to the cloud, without revealing the content of outsourced data. As such, the membership revocation mechanism brings additional computation overhead. However, CloudaSec defines a new revocation system based on (Burmester and Desmedt, 2005), without updating the secret keys of the remaining group members, in order to minimize the complexity of key management. That is, our design conveys performance advantages for large scale sharing groups.

In addition, several storage systems are based on the proxy re-encryption algorithms, in order to achieve fine grained access control \{(Xiong et al., 2012; ?),(Ateniese et al., )\}. When a recipient wants to retrieve outsourced data from the depositor, he has first to ask the cloud server to re-encrypt data file using its public key and the public master key, while considering the granted privileges. Ateniese et al. (Ateniese et al., ) propose a proxy re-encryption scheme to secure distributed storage systems and achieve efficient access control among dynamic groups. However, a collision attack between the untrusted storage server and a revoked group member can be launched, which enables to learn the decryption keys of all encrypted blocks. In (Xiong et al., 2012), the authors design an end to end content confidentiality protection mechanism for large scale data storage and distribution. They include many cryptographic mechanisms, namely the proxy re-encryption and broadcast 
revocation. Unfortunately, the subscription of a new user or the revocation of a group member requires the update of the entire group with new parameters and secret keys. That is, the complexity of user participation and revocation in their approach is linearly increasing with the number of data owners and the number of revoked users, respectively. To mitigate to such concern, CloudaSec presents a flexible revocation procedure, while considering a restricted member list, adapted to resource constrained devices.

Recently, in order to achieve efficient membership revocation system, (Liu et al., 2013) adopts a group signature mechanism. They propose a multi-owner data sharing scheme, MONA, for dynamic groups in the cloud, while preserving identity privacy from untrusted servers. Nevertheless, MONA brings an extra storage overhead at both the cloud and the group manager side, for each outsourced data file.

In (Seo et al., 2013), Seo et al. propose an improved mediated certificateless approach, in order to secure data sharing in cloud servers. In fact, the basic concept of mediated cryptography is the usage of a security mediator (SEM) which can control security capabilities for the participating entities. Once the SEM is notified that a group member is revoked, it can immediately stop the user scenario. Unfortunately, similarly to a proxy re-encryption scheme, this approach involves a trusted third party, in order to generate the partially decrypting keys. That is, it requires additional storage capacities and computation cost overhead, while considering flexible user management mechanisms.

\section{CONCLUSIONS}

The growing need for secure cloud sharing services and the attractive properties of the convergent cryptography lead us to combine them, thus, defining an innovative solution to the data outsourcing security and efficiency issues. In this paper, we design a secure data sharing scheme CloudaSec, for dynamic groups in untrusted cloud storage environments. Our approach ensures the confidentiality of outsourced data in public untrusted cloud servers and defines a smooth group revocation mechanisms. That is, flexible access control policies are enforced among users belonging to separate groups with different privileges.

Our experimental results show the efficiency of CloudaSec in scalable data sharing, while considering the impact of the cryptographic operations at the client side.

\section{REFERENCES}

https://github.com/openstack/swift.

Ateniese, G., Fu, K., Green, M., and Hohenberger, S. Improved proxy re-encryption schemes with applications to secure distributed storage. ACM Trans. Inf. Syst. Secur, 9:1-30.

Ben, L. (2007). On the implementation of pairing-based cryptosystems.

Boneh, D. and Boyen, X. (2006). On the impossibility of efficiently combining collision resistant hash functions. In In Proc. Crypto 06, pages 570-583.

Burmester, M. and Desmedt, Y. (2005). A secure and scalable group key exchange system. Inf. Process. Lett., 94(3).

Dierks, T. and Rescorla, E. (2008). RFC 5246 - The Transport Layer Security (TLS) Protocol Version 1.2. Technical report.

et al., T. G. (2002). GNU multiple precision arithmetic library 4.1.2

Fugkeaw, S. (2012). Achieving privacy and security in multi-owner data outsourcing. pages 239-244. IEEE.

Gantz, B. J. and Reinsel, D. (2012). The digital universe in 2020: Big data, bigger digital shadows, and biggest growth in the far east. IDC iView, (December):1-16.

Goyal, V., Pandey, O., Sahai, A., and Waters, B. (2006). Attribute-based encryption for fine-grained access control of encrypted data. In Proceedings of the 13th ACM conference on Computer and communications security, CCS '06, pages 89-98. ACM.

Liu, X., Zhang, Y., Wang, B., and Yan, J. (2013). Mona: Secure multi-owner data sharing for dynamic groups in the cloud. IEEE Trans. Parallel Distrib. Syst., 24(6).

Ratna, D., Rana, B., and Palash, S. (2004). Pairing-based cryptographic protocols : A survey.

Regan, K. W. Minimum-complexity pairing functions.

Seo, S.-H., Nabeel, M., Ding, X., and Bertino, E. (2013). An efficient certificateless encryption for secure data sharing in public clouds. IEEE Transactions on Knowledge and Data Engineering, 99:1.

The OpenSSL Project (2003).

Wang, C., guang Qin, Z., Peng, J., and Wang, J. (2010). A novel encryption scheme for data deduplication system. pages 265-269.

Xiong, H., Zhang, X., Yao, D., Wu, X., and Wen, Y. (2012). Towards end-to-end secure content storage and delivery with public cloud. CODASPY '12, pages $257-$ 266. ACM.

Yu, S., Wang, C., Ren, K., and Lou, W. (2010). Achieving secure, scalable, and fine-grained data access control in cloud computing. INFOCOM'10, pages 534-542.

Zarandioon, S., Yao, D. D., and Ganapathy, V. (2011). K2c: Cryptographic cloud storage with lazy revocation and anonymous access. In Secure Comm, volume 96, pages 59-76. Springer.

Zhou, L., Varadharajan, V., and Hitchens, M. (2011). Enforcing role-based access control for secure data storage in the cloud. Comput. J., 54. 